screened. As might be expected from an older population a higher cancer detection rate was observed, although a higher drop-out rate was also seen, even in those fit to proceed to a diagnostic test.

If participation in over 75 's continues to increase, particularly following the introduction of the new (more sensitive and easier to use) faecal immunochemical test in June 2019, it is likely to impact further on the already stretched colonoscopy services in England.

\section{P295 COLONIC STENTING THROUGHOUT THE LARGE BOWEL: DURABILITY AND SURVIVAL IN PATIENTS WITH PALLIATIVE COLON CANCER}

Samantha Baillie*, Slee Parrish, Giles Walker, Alec Engledow, David Dewar. Lewisham And Greenwich NHS Trust, London, UK

\subsection{6/gutjnl-2020-bsgcampus.369}

Introduction Colonic stenting for malignant obstruction is now established as the preferred treatment for patients without curative intent, and provides a lower rate of stoma formation. Guidelines in the UK (NICE) and Europe (ESGE) advocate stenting in left-sided obstruction, but neither recommend right-sided stenting due to limited data suggesting lower success and higher complication rates.

Methods We included all patients with malignant colonic obstruction who underwent attempted stenting, in a singlecentre, over a 10-year period until 2019. Data was analysed for immediate clinical outcomes, the durability of event-free survival, occurrence of late complications, re-interventions (restenting, surgery) and death.

Results 118 palliative colonic stent procedures were undertaken for malignant large bowel obstruction, with 90 leftsided tumours and 28 right-sided obstructing tumours proximal to the splenic flexure. The median patient age was 74 years (27-94), and 56\% were male. Clinical success was achieved in $87 \%$ and $70 \%$ respectively $(p=0.57)$. Technical failures $(\mathrm{L} \vee \mathrm{R})$ resulted from stool interference $(0 \mathrm{v} 11 \%$ cases) and inability to cross the stricture with a wire $(7.5 \mathrm{v}$ $16 \%$ cases). There were no procedural complications. Comparing post-procedure complications during the life of the stent, these occurred more frequently with left-sided stents $(25 \% \mathrm{v} 5 \%, \mathrm{p}=0.04)$. In these left-sided patients, there were 6 patients who required surgery with stoma for perforation, abscess formation, or re-obstruction. Stent migration occurred in 6 patients, 3 were successfully re-stented and 3 managed conservatively after CT identfied migration. Stent occlusion in a further 7 cases was managed by re-stenting at a median of 228 days (177 to 498 days). The only complication in a right-sided patient was a stent obstruction on day 536 requiring surgery with stoma formation, but there were no other interventions required in this group. Comparing left and right-sided stents, there was no statistical difference in event-free survival of $151 \quad \mathrm{v} 257$ days $(\mathrm{p}=0.17)$, and no difference in overall survival of $230 \mathrm{v}$ 257 days $(p=0.64)$.

Conclusions Clinical success after right-sided stenting appears lower for expected technical reasons. Durability of benefit and survival appears at least comparable to left-sided stenting. There was no evidence to support a higher complication rate and no re-intervention was required in the 12 months after stent insertion, compared to 1 in 5 in the left-sided group.
We believe stenting should be a management option for patients presenting with large bowel obstruction proximal to the splenic flexure with palliative intent.

\section{P296 APPROPRIATENESS OF INVESTIGATIONS FOR IRON DEFICIENCY ANAEMIA ON A FAST-TRACK PATHWAY: A MULTI-SITE TRAINEE-LED AUDIT}

Selena Dixon*, Keeley Fairbrass, Conchubhair Winters, Vivek Goodoory, Lucy Turner, Thomas Archer, Priya Oka, Mais Khasawneh, Isabel Carberry, Arslan Saleem, Robert Kennedy, Asif Khan, Amy Hicks, Sarah Mansoor, Anna Roskilly, Mustafa Jalal, Alhassan Ghodeif, Callum Hunter, Deloar Hoshen, Heather Parr, Chun Sin, Katrina Pekarska, Mitali Agarwal, Mary Elias, Daniel Taylor, Tiwonge Nyirenda, Yun Chew, Sonia Moteea, Muhammad Khan, Carl Lane, Nikolaos Pantzaris, Petya Spasova, Carrie-Anne Lee, Fahad Sheikh, Silma Shahama, Chinemelu Adilieje. Yorkshire and Humber Gastroenterology Udit and research Trainee network (YHGT)

\subsection{6/gutjnl-2020-bsgcampus.370}

Introduction There is increasing demand on services to investigate patients on 2 -week wait cancer pathways. Iron deficiency anaemia (IDA) is a common reason for referral. We aimed to assess adherence to National Institute of Clinical Excellence (NICE) suspected cancer guidelines and British Society of Gastroenterology (BSG) guidelines for investigation of IDA.

Methods Retrospective audit across 10 sites in Yorkshire, by a trainee research network. We included patients referred on a suspected cancer pathway with IDA in November 2018. Data on referral indications and investigations were collected. Anonymised data was pooled for descriptive analysis. Categorical variables are expressed as percentages.

Results 508 patients were included: median age 72 years (range 24-97); $55 \%$ female. 48\% were asymptomatic, $15 \%$ had weight loss, $13 \%$ change in bowel habit, $8 \%$ rectal bleeding, 6\% dyspepsia, 2\% dysphagia, 2\% melaena and $1 \%$ abdominal mass. Post-menopausal status was poorly recorded.

Haemoglobin $(\mathrm{Hb})$ was less than the laboratory reference range in $89 \%$. 54\% patients had low $\mathrm{Hb}$ and ferritin below the laboratory range (BSG definition for IDA). $64 \%$ and $44 \%$ patients had low $\mathrm{Hb}$ and ferritin $<30 \mu \mathrm{g} / \mathrm{L}$ or ferritin $<15 \mu \mathrm{g} /$ $\mathrm{L}$, respectively; $2 \%$ patients had a ferritin $30-100 \mu \mathrm{g} / \mathrm{L}$ and low iron indices and in $6 \%, \mathrm{Hb}$ was normal with a low ferritin.

$43 \%$ patients met NICE guidelines for 2-week referral with IDA (age $>60$ yrs, age 50-60 yrs with rectal bleeding and IDA, age $<50$ yrs with rectal bleeding and IDA). Of those not meeting criteria, $10 \%$ had other indications for referral (weight loss in 8\%). Data regarding treatment with oral iron was not available.

Coeliac serology was tested in primary care in $10 \%$, secondary care in $27 \%$ and not tested in 57\%. $49 \%$ had duodenal biopsies performed ( $2 \%$ showed coeliac disease). $62 \%$ had both coeliac serology and duodenal biopsies performed. $82 \%$ did not undergo urine testing for blood or this was not recorded. Of those undergoing investigation, $72 \%$ had both upper and lower GI investigations. The most common reason for incomplete investigation was patient choice (33\%), followed by patient fitness to undergo invasive procedures.

Conclusions NICE guidelines are poorly adhered to, although the laboratory definition of IDA is debatable. Few patients undergo serological testing for coeliac disease or urinalysis; 
most undergo endoscopic investigations or have reason not to. We plan to identify areas to improve and implement change in individual sites.

\section{P297 YIELD OF INVESTIGATIONS FOR FAST-TRACK IRON DEFICIENCY ANAEMIA REFERRALS IN YORKSHIRE: A MULTI-SITE TRAINEE-LED AUDIT}

Selena Dixon*, Keeley Fairbrass, Conchubhair Winters, Vivek Goodoory, Lucy Turner, Thomas Archer, Priya Oka, Mais Khasawneh, Isabel Carberry, Arslan Saleem, Robert Kennedy, Asif Khan, Amy Hicks, Sarah Mansoor, Anna Roskilly, Mustafa Jalal, Alhassan Ghodeif, Callum Hunter, Deloar Hoshen, Heather Parr, Chun Sin, Katrina Pekarska, Mitali Agarwal, Mary Elias, Daniel Taylor, Tiwonge Nyirenda, Yun Chew, Sonia Moteea, Muhammad Khan, Carl Lane, Nikolaos Pantzaris, Petya Spasova, Carrie-Anne Lee, Fahad Shaikh, Silma Shahama, Chinemelu Adilieje. Yorkshire and Humber Gastroenterology Udit and research Trainee network (YHGT)

\subsection{6/gutjnl-2020-bsgcampus.371}

Introduction Patients with iron deficiency anaemia are commonly referred to gastroenterology for exclusion of significant pathology. Some of these patients will have been previously investigated. We aimed to ascertain how often a cause of anaemia is found and subsequent action if no cause found.

Methods Retrospective audit across 10 sites in Yorkshire, by a trainee-led research network. We included patients referred on a suspected cancer pathway with IDA in November 2018. Data on referral criteria, investigations, diagnosis and followup were collected. Anonymised data was pooled for comparative analysis in Excel and SPSS.

Results 508 patients were included: median age 72 years (range 24-97); $55 \%$ female. $48 \%$ of these patients were asymptomatic. 42 cancers $(8 \%)$ were diagnosed: 25 colorectal (5\%), 6 oesophageal/gastric (1\%), 2 renal, 1 bladder, 2 pancreatic, 2 hepatobiliary, 1 prostate, 2 lung and 1 unknown primary.

There was no correlation between patient and age and likelihood of malignancy, but an association was seen with mean Haemoglobin $(\mathrm{Hb})$ and gastrointestinal cancer diagnosis.

Other pathology was found in $33 \%$ of those investigated: erosive gastritis $8 \%$, oesophagitis $5 \%$, erosive duodenitis $1 \%$, peptic ulcer $3 \%$, gastric polyp $1 \%$, gastric antral vascular ectasia $1 \%$, angiodysplasia $1 \%$, coeliac disease $2 \%$, colonic polyp $>10 \mathrm{~mm} \mathrm{2 \%}$, colonic polyp $<10 \mathrm{~mm} 8 \%$ and inflammatory bowel disease (1\%). 22\% patients had undergone previous endoscopic investigations in the past 5 years (indication unknown).

Where no significant pathology was found, 43\% patients were discharged without clinic review, 30\% patients were discharged following discussion of results, $9 \%$ were seen again in clinic with no further investigations requested and $16 \%$ required ongoing follow-up or further investigations. Advice regarding $\mathrm{Hb}$ monitoring (with timeframe) was given in $11 \%$, vague advice was given in $19 \%$ and no advice was given to general practitioners (GPs) in 48\%.

Conclusions An expected rate of significant pathology was found. 1 in 5 patients had undergone gastrointestinal investigations previously. 1 in 3 patients were seen in clinic following normal investigations. Better advice to GPs regarding $\mathrm{Hb}$ monitoring and subsequent management is needed and may reduce unnecessary re-referrals. Further exploratory work to identify additional predictors of significant pathology is planned.

\section{P298 CHECKPOINT INHIBITOR COLITIS: INSIGHTS FROM BENCH AND BEDSIDE}

1,2,3 Tarun Gupta* ${ }^{*}{ }^{2}$ Agne Antanaviciute, ${ }^{2}$ Anna Aulicino, ${ }^{1,3}$ Vincent Cheung, ${ }^{4,5}$ Anna Olsson-Brown, ${ }^{2}$ Ms Ana Geros, ${ }^{2}$ Kaushal Parikh, ${ }^{2}$ Ms Marta Jagielowicz, ${ }^{2}$ Hannah Chen, ${ }^{4,5}$ Joseph Sacco, ${ }^{4}$ Jonathan Heseltine, ${ }^{6}$ Sreedhar Subramanian, ${ }^{6}$ Eve Fryer, ${ }^{6}$ Elena Collantes, ${ }^{5}$ Prof Punir Pirmohammed, ${ }^{3,8}$ Benjamin Fairfax, ${ }^{3,8}$ Miranda Payne, ${ }^{8}$ Mark Tuthill, ${ }^{8}$ Meenali Chitnis, ${ }^{3,8}$ Prof Andrew Protheroe, ${ }^{3,8}$ Prof Mark Middleton, ${ }^{2}$ Hashem Koohy, ${ }^{1}$ Oliver Brain, $1,2,3$ Alison Simmons. ${ }^{1}$ Translational Gastroenterology Unit, University of Oxford, Oxford, UK; ${ }^{2}$ Weatherall Institute of Molecular Medicine, University of Oxford, Oxford, UK; ${ }^{3}$ NIHR Oxford Biomedical Research Centre, Oxford, UK; ${ }^{4}$ The Clatterbridge Cancer Centre NHS Foundation Trust, Wirral, UK; ${ }^{5}$ Institute of Translational Medicine, University of Liverpool, Liverpool, UK; ${ }^{6}$ Department of Cellular Pathology, John Raddiffe Hospital, Oxford, Oxford; 'Department of Gastroenterology, Royal Liverpool University Hospital, Liverpool, UK; ${ }^{8}$ Oxford Cancer and Haematology Centre, Oxford, UK

\subsection{6/gutjnl-2020-bsgcampus.372}

Aims Immune checkpoint blockade (ICB) is the mainstay of treatment for metastatic melanoma and lung adenocarcinoma, and their use is growing in cancer. Immune checkpoint blockade induced colitis (ICB colitis) presents a management challenge and its mechanisms remain poorly elucidated.

Methods We performed next generation single-cell RNA sequencing of immune cells from patients given ICB. We validated findings using confocal microscopy, drawing comparisons bioinformatically with ulcerative colitis. In parallel, we conducted a review of 1,074 patients given checkpoint inhibitors across two tertiary centres between 2011-2018 to discover patterns in incidence and predictors of clinical outcome.

Results Using single-cell RNA sequencing, we discovered excessive local CD8 $\mathrm{T}$ cell proliferation was a key feature of ICB colitis, and we were able to visualise higher numbers of replicating CD8 T cells in gut tissue sections of patients with colitis than those without ICB colitis. The degree of replication was greater than seen in ulcerative colitis by bioinformatic analysis.

From our clinical review, age, gender and smoking status did not alter the risk of developing colitis, whereas type of immunotherapy did (incidence 9\% in PD-1 Monotherapy vs $32 \%$ Combination Therapy). Having prior IBD did not guarantee the development of ICB Colitis. Systemic markers of inflammation (C-Reactive Protein, Albumin) did not predict outcome, whereas local markers of inflammation (endoscopic UCEIS scoring, histological Nancy Index) did.

Conclusions We putatively link novel insights from bench science to clinical trends. ICB colitis may be driven more by a gut localised inflammation response in comparison to ulcerative colitis. As we also demonstrate, this may explain why endoscopic and histological scoring may have better prognostic value than systemic measures of inflammation.

\section{P299 EVALUATION OF MOLECULAR CHARACTERISTICS OF EARLY ONSET COLORECTAL CANCER IN A POPULATION- BASED COHORT STUDY}

${ }^{1}$ Ashleigh Hamilton ${ }^{*}$, 2,3,4,5 Jacqueline A James, 2,4,5 Manuel Salto-Tellez, ${ }^{2,3}$ Stephen McQuaid, ${ }^{1}$ Ronan Gray, 1,2,5Maurice Loughrey, ${ }^{1,2}$ Helen Coleman. ${ }^{1}$ Cancer Epidemiology Research Group, Queen's University, Belfast, UK; ${ }^{2}$ Patrick G Johnston Centre for Cancer Research, Queen's University, Belfast, UK; ${ }^{3}$ Northern Ireland Biobank, Queen's University, Belfast, UK; ${ }^{4}$ Precision Medicine Centre of Excellence, Queen's University, Belfast, UK; ${ }^{5}$ Department of Cellular Pathology, Belfast Health and Social Care Trust, Belfast, UK

10.1136/gutjnl-2020-bsgcampus.373 